\title{
Photon Counting Linear Discriminant Analysis with Integral Imaging for Occluded Target Recognition
}

\author{
Seokwon Yeom* \\ School of Computer and Communication Engineering, Daegu University, Gyeongsan, Gyeongbuk \\ 712-714 Republic of Korea \\ Bahram Javidi \\ Department of Electrical and Computer Engineering, University of Connecticut, Storrs, CT USA 06269
}

(Received April 24, 2008 : revised June 2, 2008 : accepted June 3, 2008)

\begin{abstract}
This paper discusses a photon-counting linear discriminant analysis (LDA) with computational integral imaging (II). The computational II method reconstructs three-dimensional (3D) objects on the reconstruction planes located at arbitrary depth-levels. A maximum likelihood estimation (MLE) can be used to estimate the Poisson parameters of photon counts in the reconstruction space. The photon-counting LDA combined with the computational II method is developed in order to classify partially occluded objects with photon-limited images. Unknown targets are classified with the estimated Poisson parameters while reconstructed irradiance images are trained. It is shown that a low number of photons are sufficient to classify occluded objects with the proposed method.
\end{abstract}

Keyword: photon counting recognition, photon counting linear discriminant analysis (LDA), integral imaging, computational reconstruction, occluded target recognition, pattern classification.

OCIS codes : (100.6890) Three-dimensional image processing; (100.3010) Image reconstruction techniques; (030.5260) Photon counting; (100.5010) Pattern recognition and feature extraction; (040.3780) Low light level

\section{INTRODUCTION}

Integral imaging (II) records and reconstructs objects with a lenslet array [1-3]. A number of small convex lenses on the same plane record three-dimensional (3D) information on a set of elemental images. The computational reconstruction method visualizes 3D information by generating two-dimensional (2D) sectional images at arbitrary depth levels $[4,5]$. The II system has been used for the object recognition due to its capability to capture and reconstruct 3D information [6-8].

The imaging system with a photon-counting detector has potential for detection and recognition of objects at a low light level. Generally, the photon-counting imaging system requires less received power than the conventional imaging system. Also, fast processing is possible with a low number of photons. Photon-counting

*Corresponding author: yeom@daegu.ac.kr imaging has a broad range of applications such as night vision, laser radar imaging, and stellar imaging [9-13]. Automatic target recognition (ATR) at a low light level has been researched in [9-11, 14-16]. Photon-counting nonlinear matched filtering and photon-counting linear discriminant analysis (LDA) have been proposed in [14] and [15], respectively. Photon-counting scene reconstruction by maximum likelihood estimation (MLE) has been proposed in [16]. The photon counting research has been extended to reconstruction space to recognize partially occluded targets $[16,17]$.

In this paper, we propose the photon-counting linear discriminant analysis (LDA) combined with computational II in order to classify partially occluded targets. The photon-counting LDA has the same optimal criterion with Fisher's LDA [18] but the photon-counting LDA deals with photon-limited images as unknown input images and irradiance images as training images. Captured reference objects are reconstructed in 3D space and 
reconstructed images are trained to compose a decision rule of the classifier. Photon-counting scenes at arbitrary depth levels are reconstructed by the MLE of Poisson parameters [16]. The reconstructed photo-scenes are used to classify unknown targets. We show that the proposed photon-counting LDA method can handle the high dimensionality of the reconstruction space without any preprocessing of dimensionality reduction. Classification performance is analyzed in term of correct recognition rates and false acceptance rates. It will be shown that a small number of photons can classify occluded targets with the proposed method. In [16], the correlationbased scheme with computational II has been researched for the recognition of occluded targets. It is noted that one reference is considered in the matched filtering while the classifier in this paper deals with multiple hypotheses (classes) of regionally segmented objects.

The organization of the paper is as follows. In Sec. 2 , we discuss the computational reconstruction method using II for irradiance scenes and photon-counting scenes. The photon-counting LDA on the reconstruction space is discussed in Sec. 3. Photon-limited images are simulated from experimentally-derived irradiance information. In Sec. 4, these simulated images are tested for the occluded object recognition. Conclusions follow in Sec. 5 .

\section{RECONSTRUCTION WITH THE COMPUTATIONAL II METHOD}

In this section we discuss the reconstruction of irradiance information and the estimation of photon-counting parameters [16]. This reconstruction method can reduce the partial occlusion for the target visualization and recognition.

\section{A. Reconstruction of irradiance information}

The II system generates an elemental image array using a lenslet array as shown in Fig. 1(a). Each elemental image is projected through a corresponding virtual pinhole to the reconstruction plane in the computational II method [4,5]. Suppose that a point $A$ on the 3D

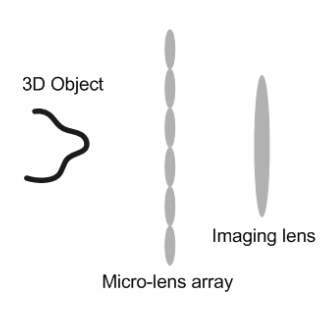

(a)

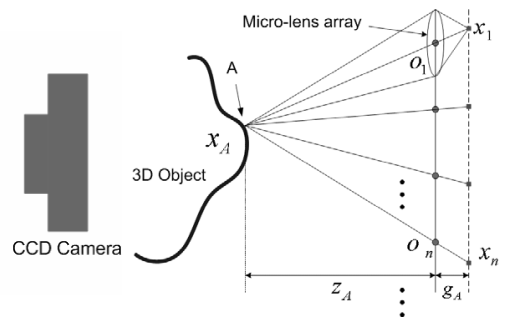

(b)
FIG. 1. (a) II pick-up system, (b) computational reconstruction model. object surface as shown in Fig. 1(b). The power density at the point $A$ is denoted as $x_{A}$. Let $x_{n}$ be the captured irradiance corresponding to the point $A$ through the $n$-th lenslet. The detection area for $x_{n}$ on the image plane is assumed to have a unit area, $o_{n}$ is the center of the $n$-th lenslet. Under the assumption that the distance $z_{A}$ between the point $A$ and the lenslet array is large enough, the same power is transferred from $x_{A}$ and collected as $x_{1}, \cdots, x_{N_{A}}$, thus, the scale factors between $x_{A}$ and $x_{n}, n=1, \cdots, N_{A}$ are assumed to be the same. Therefore, $x_{A}$ can be reconstructed by the average of $x_{1}, \cdots, x_{N_{A}}$ with a normalized scale factor:

$$
\hat{x}_{A}=\frac{1}{N_{A}} \frac{\sum_{n=1}^{N_{A}} x_{n}}{\sum_{m=1}^{N_{T}} x(m)},
$$

where $N_{T}$ is the total number of pixels in the elemental image array [16]. The pixels on the image plane projected to the nearest point to the voxel point $A$ are considered the corresponding pixels in Eq. (1). This averaging method has been newly proposed in [16] while the summation after the projection is considered in $[4,5]$. It is noted that $N_{A}$ becomes a constant which is the total number of elemental images when the distance becomes large enough for the viewing angles of all of the lenslet to cover the object surface.

\section{B. Estimation of photon-counting parameters}

Assuming the fluctuations in irradiance are small compared to the fluctuations produced by the quantized nature of the radiation, the Poisson distribution is considered for the photon-counting model [19]. In this case, the Poisson parameter for photon-counts at each pixel is assumed to be proportional to the irradiance of the pixel in the detector [9]. Therefore, the probability of a photon event at pixel $m$ can be given by

$$
\begin{aligned}
& P_{d}(y(m) ; \lambda(m))=\frac{\lambda(m)^{y(m)} e^{-\lambda(m)}}{y(m) !}, y(m)=0,1,2, \ldots, \\
& \lambda(m)=N_{P}\left[x(m) / \sum_{m=1}^{N_{T}} x(m)\right],
\end{aligned}
$$

where $y(m)$ is the number of photons detected at pixel $m, N_{P}$ is an expected number of photo-counts in the elemental image array, and $x(m)$ is irradiance at pixel $m$. Let $y_{n}, n=1, \cdots, N_{A}$ be photon counts detected with the parameter $\lambda_{n}$ which is associated with $x_{n}$. Let us consider $\lambda_{A}$ to be associated with $x_{A}$. With the assumption that $x_{1}, \cdots, x_{N_{A}}$ are the measurements of $x_{A}, \lambda_{1}, \cdots, \lambda_{N_{A}}$ are also originated from $\lambda_{A}$. Since $y_{1}, \cdots, y_{N_{A}}$ are independent Poisson random numbers with the Poisson parameter 
$\lambda_{A}$, the joint probability distribution function of photon counts is calculated as

$$
P_{d}\left(y_{1}, \ldots, y_{N_{A}} ; \lambda_{A}\right)=\prod_{n=1}^{N_{A}} \frac{\lambda_{A}^{y_{n}} e^{-\lambda_{A}}}{y_{n} !}
$$

The MLE [20] of $\lambda_{A}$ is derived as

$$
\hat{\lambda}_{A}=\frac{1}{N_{A}} \sum_{n=1}^{N_{A}} y_{n}
$$

The photon-counting parameter is estimated by the MLE (maximum likelihood estimation) of which the solution is merely the sample mean of the photon counts $[16]$.

\section{PHOTON COUNTING LDA IN THE RECONSTRUCTION SPACE}

In the photon-counting LDA combined with computational II, the reconstructed irradiance images of reference targets are trained and the class is decided by the estimated Poisson parameters. From Eq. (2) and (3), it can be shown that

$$
y_{n} \sim \operatorname{Poisson}\left(N_{p} x_{n}^{\prime}\right),
$$

where $x_{n}^{\prime}=x_{n} / \sum_{m=1}^{N_{T}} x(m)$. We derive the following relationship since the sum of independent Poisson random numbers is also a Poisson random number:

$$
N_{A} \hat{\lambda}_{A} \sim \operatorname{Poisson}\left(N_{p} N_{A} \hat{x}_{A}\right) .
$$

Eq. (7) is extended to the Poisson random vector of which parameters are proportional to the reconstructed irradiance on the reconstruction plane:

$$
\mathbf{r} \sim \operatorname{Poisson}\left(N_{p} \mathbf{0}\right),
$$

where $\mathbf{r}=\left[N_{1} \hat{\lambda}_{1} \cdots N_{v} \hat{\lambda}_{N_{v}}\right]^{\mathrm{t}}, \mathbf{o}=\left[N_{1} \hat{x}_{1} \cdots N_{v} \hat{x}_{N_{v}}\right]^{\mathrm{t}}, N_{1}, \cdots, N_{v}$ are equivalent with $N_{A}$ in Eq. (1) and (5), and the subscript $v$ is the number of voxels on the reconstruction plane.

The photon-counting LDA is derived in the reconstruction space using $\mathbf{r}$ and $\mathrm{o}$. The within-class covariance matrix of $r$ is defined as

$$
\sum_{r r}^{W}=E_{j}\left\{E_{r \mid j}\left[\left(\mathbf{r}-\boldsymbol{\mu}_{r \mid j}\right)\left(\mathbf{r}-\boldsymbol{\mu}_{r \mid j}\right)^{t} \mid j\right]\right\},
$$

and the between-class covariance matrix as

$$
\sum_{r r}^{B}=E_{j}\left[\left(\boldsymbol{\mu}_{r \mid j}-\boldsymbol{\mu}_{r}\right)\left(\boldsymbol{\mu}_{r \mid j}-\boldsymbol{\mu}_{r}\right)^{t}\right],
$$

where $E$ denotes the expectation operator, $j$ is the index of a class, $t$ denotes the matrix transpose, $\boldsymbol{\mu}_{r \mid j}=E_{r \mid j}(\mathbf{r} \mid j)$, and $\boldsymbol{\mu}_{r}=E(\mathbf{r})$. It has been shown that the projection matrix for the photon-counting LDA is obtained by the following criterion [15]:

$$
W_{P}=\max \frac{\left|W^{t} \Sigma_{r r}^{B} W\right|}{\left|W^{t} \Sigma_{r r}^{W} W\right|}=\max \frac{\left|W^{t} \Sigma_{o o}^{B} W\right|}{\left|W^{t}\left[\operatorname{diag}\left(\boldsymbol{\mu}_{o}\right) / N_{p}+\Sigma_{o o}^{W}\right] W\right|},
$$

where $\Sigma_{o o}^{B}$ is the between-class covariance matrix of $\mathbf{o}$, $\Sigma_{o o}^{W}$ is the within-class covariance matrix, and $\boldsymbol{\mu}_{o}$ is the mean vector. The column vectors of $W_{P}$ are the eigenvectors of $\left(\operatorname{diag}\left(\boldsymbol{\mu}_{o}\right) / N_{p}+\Sigma_{o o}^{W}\right)^{-1} \Sigma_{o o}^{B}$ corresponding to the non-zero eigenvalues of $\left(\operatorname{diag}\left(\boldsymbol{\mu}_{o}\right) / N_{p}+\Sigma_{o o}^{W}\right)^{-1} \Sigma_{o o}^{B}$. The rank of $\Sigma_{r r}^{B}$ is the same with that of $\Sigma_{o o}^{B}$, which is $\operatorname{rank}\left(\sum_{o o}^{B}\right) \leq \min \left[n_{c}-1, v\right]$, where $n_{c}$ is the number of classes. Therefore, $W_{P}$ is composed of at most $n_{c}-1$ orthogonal vectors when $v$ is larger than $n_{c}-1$. The maximum value of Eq. (11), $\left|W_{P}^{t} \Sigma_{r r}^{B} W_{P}\right| /\left|W_{P}^{t} \Sigma_{r r}^{W} W_{P}\right|$ is equal to the summation of non-zero eigenvalues of $\left(\operatorname{diag}\left(\boldsymbol{\mu}_{o}\right) / N_{p}+\Sigma_{o o}^{W}\right)^{-1} \Sigma_{o o}^{B}$.

It is noted that $\Sigma_{r r}^{W}$ is nonsingular with the non-zero components of $\mu_{0}$, therefore, the photon-counting LDA does not suffer from the singularity problem during the training, which is inevitable when the number of training images is smaller than the dimensionality of o [15].

The following Euclidean distance is a metric for decision making:

$$
\hat{j}=\underset{j=1, \ldots, n_{c}}{\arg \min }\left\|W_{P}^{t}\left(\mathbf{r}_{\text {test }}-N_{p} \boldsymbol{\mu}_{o \mid j}\right)\right\|,
$$

where $n_{c}$ is the number of classes, $\mathbf{r}_{\text {test }}$ is the reconstructed photon-counting vector for unknown targets, and $\boldsymbol{\mu}_{o \mid j}$ is the class-conditional mean vector of $\mathbf{o}$, which is obtained during the training process.

\section{EXPERIMENTAL AND SIMULATION RESULTS}

In this paper The II pick-up system is composed of a lenslet array and a pick-up camera. Except for a magnification factor and negligible aberration of the imaging lens in the pick-up camera, the image on the image plane of the lenslet array and the captured image by the CCD camera are assumed to be identical. Four sets of elemental images have been obtained from two toy cars without or with occlusion. The pitch of each lenslet is $1.09 \mathrm{~mm}$ and the focal length of each lenslet is about $3 \mathrm{~mm}$. The size of the car is about 4.5 $\mathrm{cm} \times 2.5 \mathrm{~cm} \times 2.5 \mathrm{~cm}$. The distance between the imaging lens and the lenslet array is $9.5 \mathrm{~cm}$, the distance 


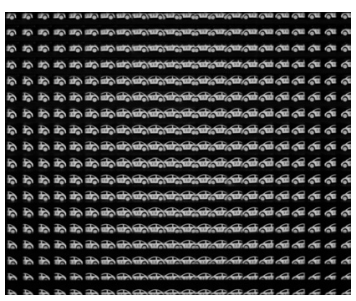

(a)

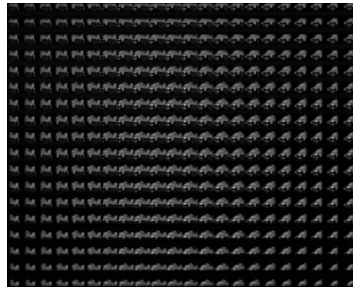

(c)

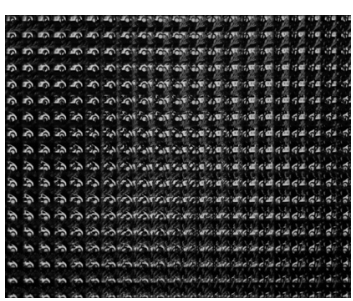

(b)

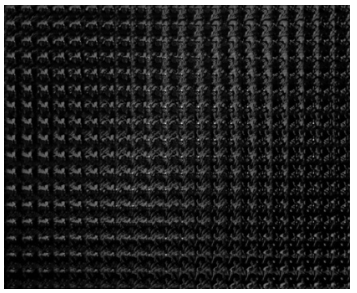

(d)
FIG. 2. Elemental image arrays, (a) car 1, (b) occluded car 1, (c) car 2, (d) occluded car 2.
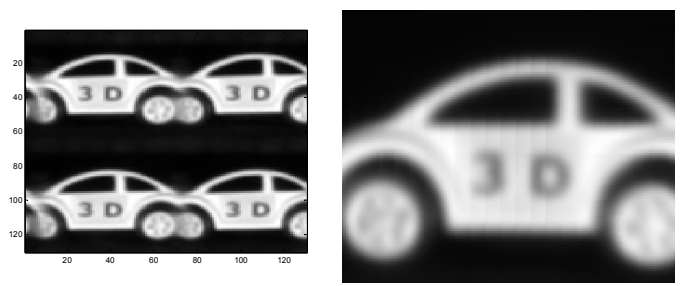

(a)
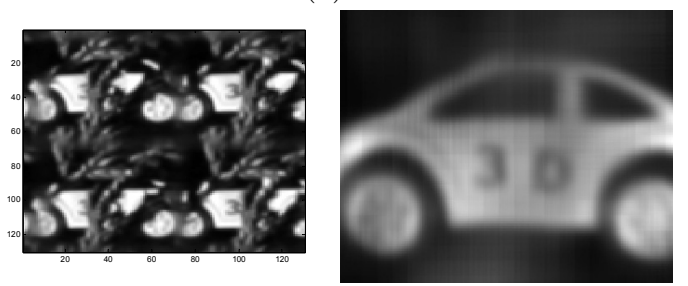

(b)

FIG. 3. Reconstructed car 1 at $74 \mathrm{~mm}$, (a) central part of FIG. 2(a) and reconstructed image, (b) central part of FIG. 2(b) and reconstructed image.

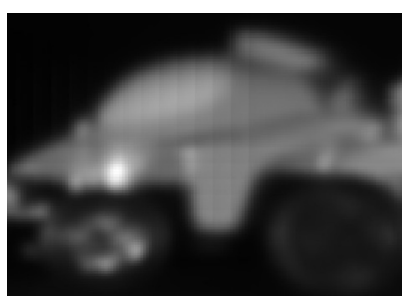

(a)

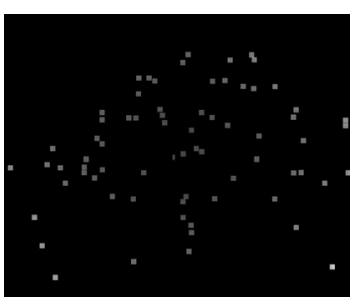

(b)
FIG. 4. Computational reconstruction of car 2 at 74 $\mathrm{mm}$, (a) irradiance, (b) photon counting parameters with $N_{p}=100$.

between the lenslet array and the occluding object is $4 \sim 5 \mathrm{~cm}$, the distance between the lenslet array and the toy car is $9.5 \mathrm{~cm}$. To simulate the partial occlusion, a
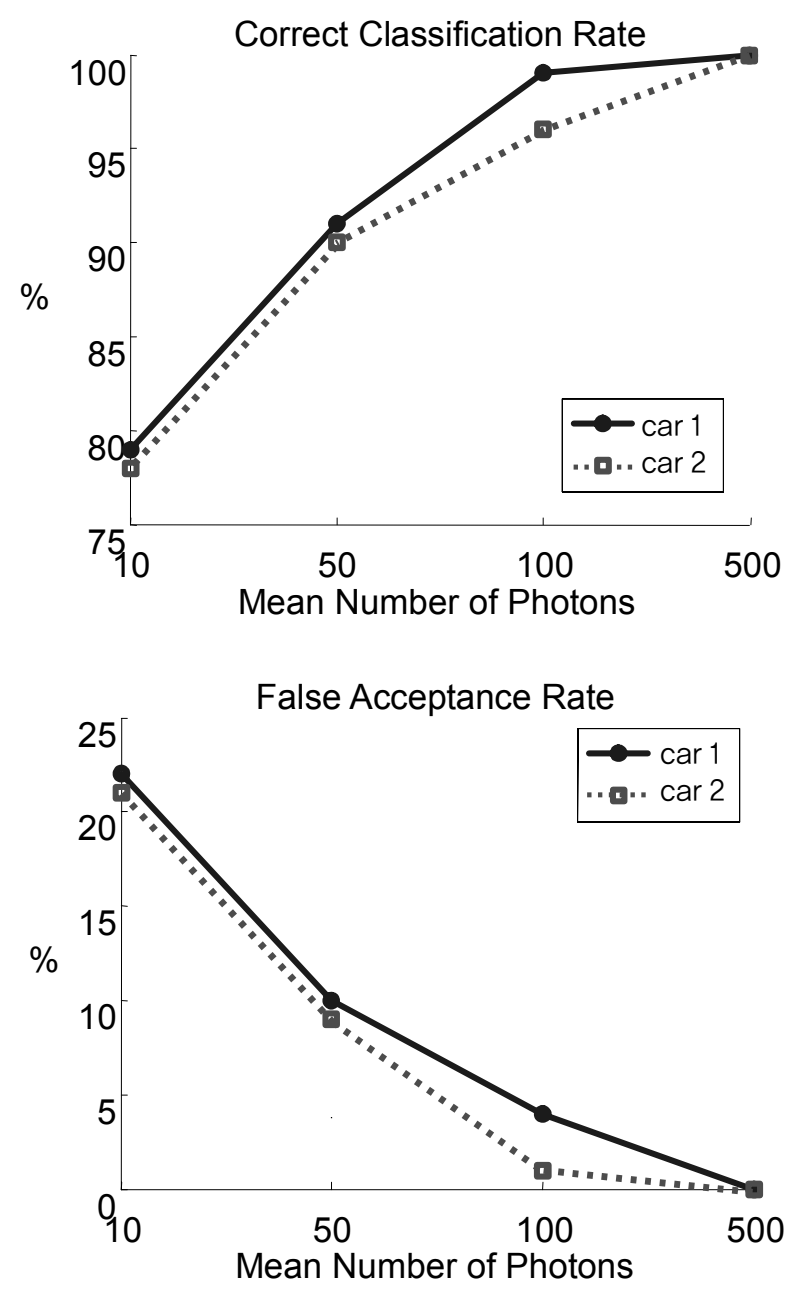

FIG. 5. Recognition performance, (a) correct classification rates, (b) false acceptance rates.

tree model is placed between the toy car and the lensletarray. Each elemental image array is composed of $1419 \times 1161$ pixels and the number of elemental images in the array is $22 \times 18$. Figure 2 show the elemental image arrays of car 1 (class 1 ) and car 2 (class 2) without or with the partial occlusion.

Figure 3(a) and 3(b) show the reconstructed images of car 1 without occlusion and with occlusion, respectively. For testing, we simulate 100 photon-limited elemental image arrays, each with a random number of photons following the Poisson distribution. Photon-counting parameters are estimated at depth of $74 \mathrm{~mm}$ for each of photon-limited elemental image array. Figure 4(a) and $4(\mathrm{~b})$ show the reconstructed image and the estimated photon-counting parameters of car 2, respectively. Figure $5(\mathrm{a})$ and 5(b)show the recognition results: classification rates and false acceptance rates varying the average number of photons, respectively. The photon-counting LDA with computational II is shown to be a robust recognition scheme for occluded targets showing promising results even at a very low level of photons. It is clear 
that the recognition performance is highly related with the quality and quantity of "true" information of targets in interest. The quantity of the information depends on the number of photons detected and the quality on the occlusion effect. We leave a thorough analysis of the system performance regarding the occlusion and the photon number for the future research topic.

\section{CONCLUSIONS}

In this paper, we propose a classification method for partially occluded targets with photon-counting LDA and computational II. The occluded object information is reconstructed in 3D space with computational II. The photon-counting LDA satisfies the optimal criterion function in the application of photon-limited images. It is shown that a small number of photons are sufficient to classify occluded targets with the proposed method.

\section{ACKNOWLEDGEMENT}

This research was supported by the Daegu University Research Grant, 2007.

\section{REFERENCES}

[1] G. Lippmann, "La photographie integrale," C. $R$ Acad. Sci. vol. 146, no. 139, pp. 446-451, 1908.

[2] J.-S. Jang and B. Javidi, "Time-multiplexed integral imaging for 3D sensing and display," Optics and Photonics News, vol. 15, no. 4, pp. 36-43, 2004.

[3] J.-Y. Song, B. Javidi, and K.-D, Kwack, "Methods for displaying three-dimensional images," Proceedings of the IEEE, vol. 94, no. 3, pp. 502-523, 2006.

[4] S.-H. Hong, J.-S. Jang, and B. Javidi, "Three-dimensional volumetric object reconstruction using computational integral imaging," Optics Express, vol. 12, no. 3, pp. 483-491, 2004.

[5] Y. S. Hwang, S. H. Hong, and B. Javidi, "Free view 3 -D visualization of occluded objects by using computational synthetic aperture integral imaging," Journal of Display Technology, vol. 3, no. 1, pp. 64-70, 2007.

[6] B. Javidi, R. Ponce-Diaz, and S. H. Hong, "Threedimensional recognition of occluded objects by using computational integral imaging," Optics Letter, vol. 31, no. 8, pp. 1106-1108, 2006.

[7] J.-H. Park, J. Kim, and B. Lee, "Three-dimensional optical correlator using a sub-image array," Optics Express, vol. 13, no. 13, pp. 5116-5126, 2005.

[8] S. Yeom and B. Javidi, "Three-dimensional distortion tolerant object recognition using integral imaging," Optics Express, vol. 12, no. 23, pp. 5795-5809, 2004.

[9] G. M. Morris, "Scene matching using photon-limited images," Journal of Optical Society of America A, vol. 1, no. 5, pp. 482-488, 1984.

[10] E. Watson and G. M. Morris, "Comparison of infrared upconversion methods for photon-limited imaging," Journal of Applied Physics, vol. 67, no. 10, pp. 60756084, 1990.

[11] E. Watson and G. M. Morris, "Imaging thermal objects with photon-counting detector," Applied Optics, vol. 31, no. 23, pp. 4751-4757, 1992.

[12] L. Duraffourg, J.-M. Merolla, J.-P. Goedgebuer, N. Butterlin, and W. Rhods, "Photon counting in the 1540-nm wavelength region with a Germanium avalanche photodiode," IEEE Journal of Quantum Electronics, vol. 37, no. 1, pp. 75-79, 2001.

[13] M. Guillanume, P. Melon, and P. Refregier, "Maximumlikelihood estimation of an astronomical image from a sequence at low photon levels," Journal of Optical Society of America A, vol. 15, no. 11, pp. 2841-2848, 1998.

[14] S. Yeom, B. Javidi, and E. Watson, "Photon counting passive 3D image sensing for automatic target recognition," Optics Express, vol. 13, no. 23, pp. 9310-9330, 2005.

[15] S. Yeom, B. Javidi, and E. Watson, "Three-dimensional distortion-tolerant object recognition using photoncounting integral imaging," Optics Express, vol. 15, no. 4, pp. 1513-1533, 2007.

[16] S. Yeom, B. Javidi, C.-W. Lee, and E. Watson, "Photoncounting passive 3D image sensing for reconstruction and recognition of partially occluded objects," Optics Express, vol. 15, no. 24, pp. 16189-16195, 2007.

[17] S. Yeom, "Photon-counting analysis with computational integral imaging for occluded target recognition(invited paper)”, 15th the Optoelectronics and Optical Communications Conference, 2008.

[18] R. O. Duda, P. E. Hart, and D. G. Stork, Pattern classification 2nd, (Wiley Inter-science, New York, 2001).

[19] J. W. Goodman, Statistical Optics, (John Wiley \& Sons, Inc. 1985).

[20] A. Papoulis, Probability, Random Variables, and Stochastic Processes $3^{\text {rd }}$ ed. (McGraw-Hill, Inc. 1991). 